\title{
Wide-Area Haptic Guidance: Taking the User by the Hand
}

\author{
Antonia Pérez Arias and Uwe D. Hanebeck
}

\begin{abstract}
In this paper, we present a novel use of haptic information in extended range telepresence, the wide-area haptic guidance. It consists of force and position signals applied to the user's hand in order to improve safety, accuracy, and speed in some telepresent tasks. Wide-area haptic guidance assists the user in reaching a desired position in a remote environment of arbitrary size without degrading the feeling of presence. Several methods for haptic guidance are analyzed. With active haptic guidance, the user is guided by superimposed forces that pull him into the desired direction of motion, whereas under passive haptic guidance, the movement of the user is lightened in the preferred direction and constrained in the other directions. By using closed-loop haptic guidance instead of open-loop haptic guidance, not only is the user guided to his target but also the deviation from the desired target path is reduced. The proposed guidance methods were tested with a haptic interface specifically designed for extended range telepresence.
\end{abstract}

\section{INTRODUCTION}

Already today, robots are sent to places not accessible for humans, be it for safety reasons or convenience. Examples of those scenarios include exploration of wild territories or foreign planets and nuclear cleanup in contaminated areas. Those scenarios usually require the surveillance and the intervention of a human operator who teleoperates the robot and an intuitive user interface that facilitates his work.

Extended range telepresence provides an intuitive way to explore remote environments. The feeling of presence is achieved by visual and acoustic sensory information recorded from the target environment and presented to the user on an immersive display. In order to use the sense of motion as well, which is specially important for human navigation and path finding, the user's motion is tracked and transferred to the teleoperator, e.g., a mobile robot, in the target environment. As a result, in extended range telepresence the operator can additionally use the proprioception, i.e., the sense of motion, to navigate the teleoperator by natural walking, instead of using devices like joysticks, pedals, or steering wheels.

Without further processing of the motion information, the motion of the operator is restricted to the size of the user environment, which is limited, for example, by the range of the tracking system or the available space. Motion Compression [1] solves this problem by mapping the desired path in the target environment to a feasible path in the user environment by minimizing proprioceptive and visual inconsistencies.

Antonia Pérez Arias and Uwe D. Hanebeck are with the Intelligent Sensor-Actuator-Systems Laboratory (ISAS), Institute for Anthropomatics, Karlsruhe Institute of Technology (KIT), Karlsruhe, Germany, antonia.perez@kit.edu, uwe.hanebeck@ieee.org
A system such as the one described above allows telepresent exploration of arbitrarily large target environments [2] and constitutes an appropriate user interface for virtual reality scenarios as well. However, in virtual reality, the user is not represented by a teleoperator but by an avatar. In the following, we use the term proxy for both teleoperators and avatars.

Haptic information from the target environment is indispensable, so that the user can perceive objects and obstacles in the target environment more realistically. For this purpose, a large haptic interface that allows for simultaneous haptic interaction and wide-area motion was developed [3]. Fig. 1 shows the user interface in our extended range telepresence system.

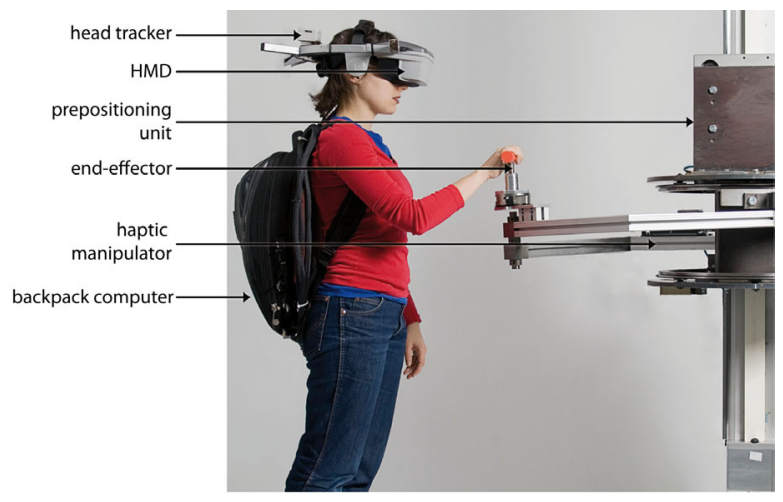

Fig. 1. User interface in the extended range telepresence system.

In some cases, it may be necessary to keep the user off certain regions in the target environment, which are dangerous for the teleoperator, or to guide the user to a desired target. We propose to use the haptic channel for presenting additional information to the user with the purpose of guiding him on a desired path in the target environment.

Furthermore, with Motion Compression, the user shows a behaviour that resembles a human walking on an inclined plane. This occurs because Motion Compression introduces small deviations in the proxy's orientation, which cannot always be compensated by the user. With wide-area haptic guidance, these deviations can be compensated as well.

Wide-area haptic guidance heavily draws from previous work on virtual fixtures [4]-[8]. [4] first presented the concept of virtual fixtures as an overlay of abstract sensory information on a workspace in order to improve the user performance in telemanipulation tasks. Virtual fixtures can be either guiding virtual fixtures or forbidden-region virtual fixtures [5]. [6] presents virtual fixtures for teleoperation 
tasks. [7] and [8] also present haptic assistance functions for a collaborative telepresence system.

Much work on virtual fixtures for cooperative systems, e.g., [9], [10], has been based on the Steady-Hand robot [11], an admittance-type cooperative manipulator designed for microsurgical procedures. It is also possible to implement virtual fixtures with passive robotic devices, such as Cobots [12]. Cobots (Collaborative Robots), typically used in assembly tasks, utilize the kinematic properties of the hardware design to create motion guidance. However, most of these haptic devices assume an immobile user and a restricted workspace. Haptic interfaces for extended range telepresence permit to extend the concept of haptic guidance in order to guide the human operator in large environments.

In this paper, we extend the concept of virtual fixtures to wide-area haptic guidance. The concept of wide-area haptic guidance is illustrated in Fig. 2. The position of the user is transformed into the target environment. A desired target path is inferred based on the user's motion, on additional information from the target environment, or on task-specific criteria. The haptic guidance augments the haptic rendering from the application with haptic cues in order to guide the user on the desired target path. Besides aiding the user, widearea haptic guidance must guarantee transparency in order to provide a high degree of immersion to the user.

In this paper, we propose two complementary guidance methods. With active guidance, a force is displayed that "pulls" the user toward the desired direction of motion, whereas with passive guidance, the motion of the user is constrained along non-desired directions. The proposed control methods were implemented and assessed on the large haptic interface. In this haptic interface, the localization and the position control of the haptic manipulator are accurately performed by a large grounded cartesian prepositioning unit. Due to the grounded frame, the haptic manipulator has a high force capability, whereas the large workspace allows for wide-area motion.

Wide-area haptic guidance opens a wide variety of applications, for example, in inspection tasks in dangerous environments, virtual training of evacuations, gaming applications, etc.

The remainder of this paper is structured as follows. In Section II, the problem of guiding a user in a largescale environment is explained. Section III discusses two different solutions for guiding the user by means of haptic information. Section IV presents the concepts of open-loop and closed-loop haptic guidance and Section $\mathrm{V}$ explains how the haptic guidance works in Motion Compression. The experimental set-up is described in Section VI and experiments conducted to evaluate the guidance methods are presented in Section VII.

\section{Problem Formulation}

We assume a bilateral telepresence system, in which the position of the operator is sent to the proxy and interaction forces from the target environment are sent back to the operator. Without further processing of the position data, the

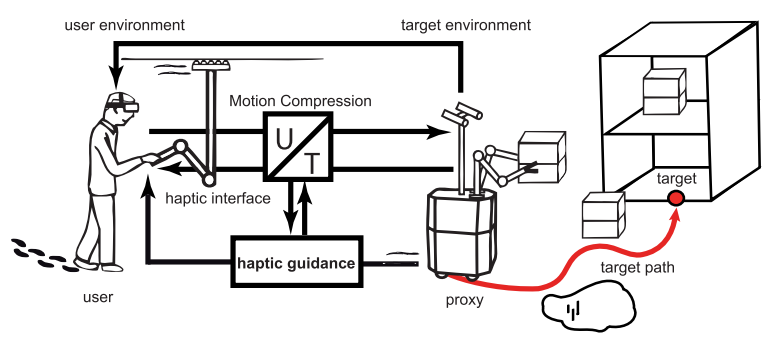

Fig. 2. Concept of wide-area haptic guidance.

motion of the operator is restricted to the size of the user environment.

Motion Compression (MC) [1] allows for wide-area motion in the target environment. To achieve this, a desired target path is first calculated based on the user's motion, on additional information from the target environment, and/or on task-specific requirements. MC transforms the desired target path into a feasible path in the user environment. Then, MC guides the user on the user path and at the same time the proxy on the target path by means of a modification of the proxy's orientation.

In many applications of telepresence, it can be necessary to keep the proxy from entering into potentially dangerous regions of the workspace, or to assist the human operator in carrying out a structured task, while maintaining a high degree of operator control.

The standard guidance provided by MC is intrinsically visual and works because the user walks goal-oriented in the direction in which he perceives the target. However, if the user must be guided either to a target that is not in his field of view or along a complicated path, the visual guidance may not be sufficient to keep the user on the desired path.

Moreover, with MC the user has to compensate for the small deviations of the proxy in order to walk on the desired target path. If the user does not turn enough, e.g., due to his own dynamics, the user is prone to leave the target path and in the worst case also the user environment.

In this work, we propose a novel wide-area haptic guidance, which adds a haptic component to the user guidance in MC. Wide-area haptic guidance consists of force and position cues applied to the user in order to guide him to a desired target and to help him walk on the desired target path.

\section{HAPTIC GUIDANCE APPROACH}

Besides a purely visual guidance, we have the possibility to influence the behavior of the user with haptic information. The goal of this section is to find adequate methods to guide the user in the target environment by means of haptic information. Two potential solutions will be discussed: actively influencing the movement of the user: active haptic guidance, or by favoring the motion of the user in the preferred direction: passive haptic guidance.

\section{A. Active Haptic Guidance}

In the first method, a guidance force is applied to the user's hand in order to correct his deviation from a desired 
path and/or to guide him to a desired target. The total force displayed to the user $\underline{F}_{r e f}$ is

$$
\underline{F}_{r e f}=\underline{F}_{G}+\underline{F}_{T},
$$

where $\underline{F}_{G}$ is the guidance force and $\underline{F}_{T}$ is the contact force from the target environment.

The guidance correction depends on additional sensor data and on knowledge about the performed task. The guidance force is, therefore, task dependent and takes the general form

$$
\underline{F}_{G}=-f\left(\underline{x}, \underline{x}_{g}\right) \frac{\underline{x}-\underline{x}_{g}}{\left\|\underline{x}-\underline{x}_{g}\right\|},
$$

where $\underline{x}$ is the position of the user and $\underline{x}_{g}$ the position of the goal. The guidance force is directed toward the desired position of the user and its magnitude $f\left(\underline{x}, \underline{x}_{g}\right)$ must be adjusted to increase the performance of the guidance by keeping the feeling of presence at the same time.

In the simplest method, a constant force could be applied in the direction of the target. The distance to the target can be taken into account, so that a larger force is applied when the distance increases. In a time-dependent approach, the force increases with time when the position of the user is outside a proximity region from the target.

\section{B. Passive Haptic Guidance}

Active haptic guidance can lead to unexpected movements of the haptic manipulator and thus deteriorate the feeling of presence. In passive user guidance, the velocity of the manipulator is proportional to the force applied by the user so that the manipulator does not move if the user does not apply any force. Similar virtual fixtures are presented in [9]. An instantaneous preferred direction $\underline{d}$ is calculated based on the position of the user relative to the desired path or goal. The applied force $\underline{F}_{\text {meas }}$ is then decomposed into one component that is the projection on the preferred direction $\underline{F}_{\|}$and the components in the non-preferred directions $\underline{F}_{\perp}$, so that $\underline{F}_{\text {meas }}=\underline{F}_{\|}+\underline{F}_{\perp}$.

By eliminating or attenuating the commanded motion due to the applied force in the non-preferred directions, we create the passive guidance along the preferred direction. The velocity control law of the manipulator can then be written in absence of contact force from the target environment as

$$
\underline{\dot{x}}_{r e f}=c_{\|} \underline{F}_{\|}+c_{\perp} \underline{F}_{\perp},
$$

where $\underline{\dot{x}}_{r e f}$ is the commanded velocity of the manipulator, and $c_{\|}$and $c_{\perp}$ are the compliances of the manipulator parallel and orthogonal to $\underline{d}$ respectively.

Hence, the commanded motion of the manipulator with interaction forces is thus proportional to an anisotropic compliance matrix $C$ and can be written as

$$
\underline{\dot{x}}_{r e f}=C\left(\underline{d}, c_{\|}, c_{\perp}\right)\left(\underline{F}_{\text {meas }}-\underline{F}_{T}\right),
$$

where $\underline{F}_{T}$ is again the contact force from the target environment.

$C$ is a time-varying compliance matrix that has large values in the instantaneous preferred direction and small or zero values in the non-preferred direction, so that the user perceives the movement in the preferred direction as easier. The largest compliance value in the preferred direction $c_{\|}$ is limited by the stability of the haptic interface. Varying the compliance in the non-preferred direction $c_{\perp}$ creates different levels of guidance, from soft in the case $0<c_{\perp}<$ $c_{\|}$to hard guidance in the case $c_{\perp}=0$. When $c_{\|}=c_{\perp}$, the compliance is isotropic and no guidance occurs. Soft guidance gives the user the freedom to move away from the path by allowing some motion in the non-preferred direction. Alternatively, with hard guidance none or almost none of the non-preferred force component is permitted, leaving the user with no or little freedom to deviate from the preferred path. A hard guidance could be necessary in order to keep the user from entering forbidden regions in the target environment.

Because in passive haptic guidance no external forces are applied, this guidance results in a more natural and transparent feeling for the user. However, the choice of the guidance method is task-dependent. Furthermore, in order to increase the velocity in the preferred direction, an external force can be used with passive guidance that steers the user toward the desired path.

\section{Path Control Methods for Haptic Guidance}

Depending on the application, it may be desirable to guide the user to a certain goal or to keep him on a reference path in the target environment. Regarding the different requirements of the haptic guidance, we can classify it in open-loop haptic guidance and closed-loop haptic guidance.

\section{A. Open-Loop Haptic Guidance}

In open-loop haptic guidance, the user is assisted in reaching his target. Only the position of the target is taken into account to calculate the guidance commands. Openloop haptic guidance is therefore adequate for targeting applications, in which the user must reach a goal but the covered path is not relevant.

In open-loop active guidance, the guidance forces are accordingly directed to the target. In open-loop passive guidance, the instantaneous preferred direction is defined based on the position of the desired target relative to the position of the user.

\section{B. Closed-Loop Haptic Guidance}

Closed-loop haptic guidance aims at keeping the user on a desired target path. Since deviations occur and are not known in advance, it is necessary to control the state of the user in order to reduce them. Therefore, the guidance commands depend on the target position, the target path, and the position of the user. Closed-loop haptic guidance is required for pathfollowing tasks.

A simple way to control the user with closed-loop active guidance is, for example, by means of guiding forces directed to the closest position on the target path whose magnitude is proportional to the deviation. Analogously, we could implement closed-loop passive guidance by making the instantaneous preferred direction point to the nearest position 
on the path, and varying the compliance in the non-preferred direction depending on the path-tracking error.

Otherwise, it might be convenient to smoothly guide the user to another position on the target path. In this case, the direction of the guiding forces (in active guidance), as well as the instantaneous preferred direction of motion (in passive guidance) are tangent to a smooth curve between the user position and the desired position on the path.

\section{Haptic Guidance AND Motion COMPRESSion}

In order to guide the user on large target paths, the desired path in the target environment is transformed by MC into a feasible path in the user environment. The nonlinear transformation is length and angle preserving only in a narrow region along the target path. The transformation does not preserve curvature, so that a straight segment in the target environment becomes a curved segment in the user environment. For this reason, the control of the user in both environments is equivalent only if deviations from the target path are small. In this case, deviations from the paths are preserved by MC and it makes no difference whether the user is guided on the target path or on the previously calculated user path. However, the deviation from the desired target path is usually not negligible.

Therefore, the direction of the guidance force and/or the instantaneous preferred direction, $\underline{d}_{T}$ are planned in the target environment based on the position of the target $\underline{x}_{G}$ and the position of the user in the target environment $\underline{x}_{E, T}$. Fig. 3 shows the control structure of the telepresence system with wide-area haptic guidance. The guidance direction $\underline{d}_{T}$ is transformed by MC into $\underline{d}_{U}$. The user is then controlled to follow this direction in the user environment with active guidance (AG), passive guidance (PG), or a combination of both. At the same time, the interaction force from the target environment $\underline{F}_{T, U}$ is presented to the user on the haptic display.

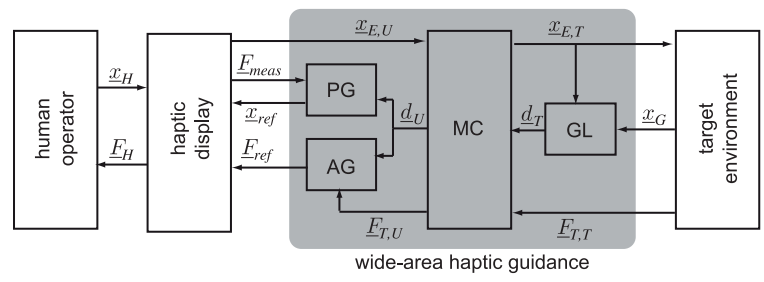

Fig. 3. Haptic interface with wide-area haptic guidance.

As a result, the user is still able to interact with the target environment while he or she is guided back on the desired path. The visual guidance of MC is essential for a high degree of immersion and a consistent guidance in largescale scenarios. Without MC, a consistent guidance is only possible in target environments that coincide with the user environment.

\section{EXPERIMENTAL SETUP}

Experiments were conducted using a large haptic interface specifically designed for extended range telepresence
(Fig. 4). The haptic interface consists of two subsystems: a linear prepositioning unit that moves with the user along the user path, and a manipulator arm attached to the prepositioning unit that is used to display defined forces at any position in the user environment. In this way, the workspace of the haptic interface covers the whole user environment. The prepositioning unit is realized as grounded portal carrier system of approximately $5 \times 5 \mathrm{~m}^{2}$. A typical motion speed of $2 \mathrm{~m} / \mathrm{s}$ and an acceleration of $2 \mathrm{~m} / \mathrm{s}^{2}$ are achieved. The manipulator arm covers the human arm workspace and has planar movement. It is implemented as a planar SCARA arm, which is attached to the portal carrier. The manipulator arm is able to display forces up to $100 \mathrm{~N}$ at the end-effector. This construction has the advantage of a high force capability and an accurate positioning of the manipulator, which is directly determined through a high resolution magnetic positioning system situated on the rails.

In our system, the haptic interface is modelled as an admittance, which transforms the reference force to be displayed into the reference motion of the end-effector. The admittance model shapes the desired dynamic behaviour of the system by compensating the natural device dynamics. The reference position of the linear prepositioning unit, which can be easily controlled in cartesian coordinates, is calculated by optimizing the manipulator's configuration. More details about the force and position control of the haptic interface can be found in [3].

For good immersion, the user wears a high-quality head mounted display with a resolution of $1280 \times 1024$ Pixels per eye. The position of the user is tracked by an acoustic tracking system, that provides approximately 20 position and orientation updates per second.

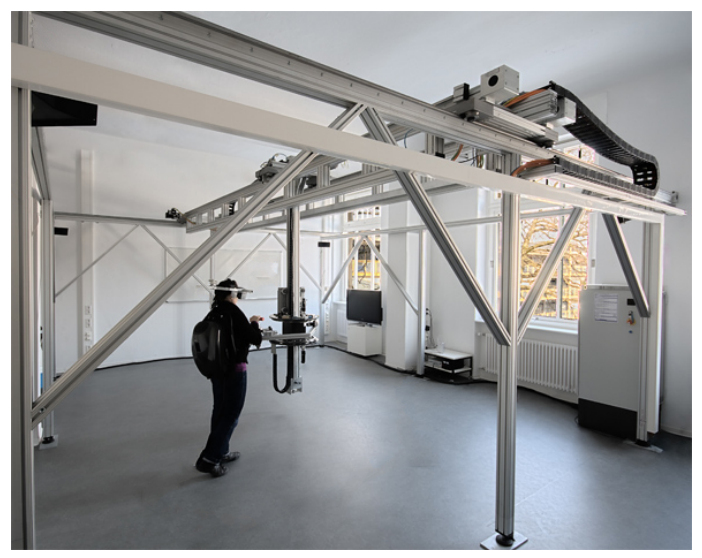

Fig. 4. Haptic interface for interaction with extended target environments.

\section{Evaluation}

\section{A. Experimental Design and Procedure}

An initial test study was performed in order to identify the most convenient guidance parameters for both, active and passive force control methods. A total of eight subjects took part in the experiment. A simple task had to be performed by each subject consisting of reaching a target position with 
open-loop haptic guidance. The subjects were told to walk without any visual information in the direction dictated by the guidance and stop as soon as they believed they had found the target. The target was placed at random positions but the distance between the target and the start position was the same, $5 \mathrm{~m}$, in all test runs. In this way, the test person cannot learn the location of the target, but the experiments can still be compared. Before data was collected, subjects familiarized themselves with the haptic interface and the task. Each subject repeated the task with four different levels of active and passive guidance, respectively. The guidance parameters presented to the subject at each test run were randomly chosen.

Active haptic guidance was implemented as guiding forces directed toward the target with $f\left(\underline{x}, \underline{x}_{q}\right)=k$. Four parameters were tested from soft to very hard guidance. Open-loop passive haptic guidance was implemented as a small damping $D_{\|}$just in the direction toward the target $\left(v_{\|}=\frac{1}{D_{\|}} f_{\|}\right)$and a large damping $D_{\perp}$ in the other directions, including the opposite to the preferred direction $\left(v_{\perp}=\frac{1}{D_{\perp}} f_{\perp}\right)$, where $v$ makes reference to the commanded motion of the endeffector and $f$ to the measured force. $D_{\|}=10 \mathrm{Ns} / \mathrm{m}$ was selected, since some damping is required to ensure the stability of the admittance control. Again, four damping coefficients were tested from soft to very hard guidance.

\section{B. Evaluation Results}

The metrics used to compare the guidance methods and the guidance parameters are: the average execution time, the average error from the target position, and both the maximum and the mean force applied by the user. The maximum applied force should be kept as small as possible in order to provide the user with a high degree of immersion. The mean of the applied force is also a measure of the energy employed by the user to perform the task. A questionnaire was also used to evaluate the subjective performance of the guidance methods. The subjects were asked which kind of guidance (active or passive) they liked most, and which level of guidance (soft, medium, hard, or very hard) was more adequate for the task in each case.

The experimental results are shown in Table I, with the average values of error, execution time, maximum applied force and mean absolute force during the targeting task under various levels of guidance.

Execution time: It is remarkable, that with active guidance the target is reached faster than with passive guidance even with a small level of guidance $k=8 \mathrm{~N}$. The positioning is faster with $k=13 \mathrm{~N}$. With passive guidance, the best execution time was achieved with $D_{\perp}=200 \mathrm{Ns} / \mathrm{m}$. These results indicate that an increase in the level of guidance does not necessarily decrease the execution time. This can be due to the fact that the user is too restricted in his or her motion.

Average Error: A certain level of haptic guidance is required to reach the target position. However, with active guidance, the best accuracy is achieved with a soft guidance $k=8 \mathrm{~N}$, whereas with passive guidance, the most accurate
TABLE I

AVERAGE VALUES OF EXECUTION TIME, ERROR, MAXIMUM APPLIED FORCE AND MEAN ABSOLUTE FORCE IN A TARGETING TASK UNDER VARIOUS LEVELS OF GUIDANCE

\begin{tabular}{|l||l|l|l|l|}
\hline Guidance level & $\begin{array}{l}\text { Time } \\
(\mathrm{sec})\end{array}$ & $\begin{array}{l}\text { Error } \\
(\mathrm{m})\end{array}$ & $\begin{array}{l}\text { Max. } \\
\text { force } \\
(\mathrm{N})\end{array}$ & $\begin{array}{l}\text { Mean } \\
\text { force } \\
(\mathrm{N})\end{array}$ \\
\hline Active & & & & \\
\hline$k=8$ & 27.755 & 0.047 & 10.053 & 7.101 \\
\hline$k=10$ & 24.721 & 0.067 & 13.228 & 8.557 \\
\hline$k=13$ & 23.392 & 0.094 & 17.480 & 10.224 \\
\hline$k=15$ & 25.043 & 0.087 & 20.756 & 14.135 \\
\hline Passive & & & & \\
\hline$D_{\perp}=75$ & 39.046 & 0.094 & 16.945 & 6.086 \\
\hline$D_{\perp}=100$ & 37.195 & 0.075 & 17.341 & 6.910 \\
\hline$D_{\perp}=200$ & 28.397 & 0.069 & 24.913 & 8.599 \\
\hline$D_{\perp}=400$ & 28.516 & 0.032 & 31.244 & 9.121 \\
\hline
\end{tabular}

positioning is achieved with the highest level of guidance $D_{\perp}=400 \mathrm{Ns} / \mathrm{m}$.

Applied force: The force and the energy applied by the user increase obviously with the level of guidance. The maximum applied force is generally larger with passive guidance, whereas the mean of the applied force increases faster with the level of active guidance.

Subjective measure: $80 \%$ of the subjects preferred the active guidance for the positioning task. These subjects reported that they preferred active guidance because it was easier to find the target than with passive guidance. With active guidance, the soft active guidance was preferred by $60 \%$ of the subjects, the hard guidance by $20 \%$, and the very hard guidance by $20 \%$. However, $80 \%$ of the subjects liked most the very hard passive guidance and $20 \%$ the hard passive guidance.

These results indicate that a small active guidance force is enough for guiding the user to a target, whereas high active forces are rather disturbing for the user. On the other hand, passive guidance leads to a high accuracy with high levels of guidance. Because no external forces are applied to the user and the energy required does not increase much with the level of guidance, passive guidance should be more transparent and safer for the user in order achieve a hard guidance. A soft passive guidance could be helpful, for example, for indicating the desired direction without forcing the user to follow it. For this reason, it would be interesting to combine both types of guidance depending on the scenario and the requirements of the task.

\section{Closed-Loop Guidance Test}

The second kind of experiments consisted in guiding a user on a large straight path in the target environment with MC. For this experiment, we used a virtual target environment modelled as a large empty plane with a single straight path of length $25 \mathrm{~m}$ drawn on the floor. The user performed the pathfollowing task with closed-loop haptic guidance (Fig. 5(a)) and without haptic guidance (only with visual guidance) (Fig. 5(b)). The same visual information from the target environment was presented to the user in both cases. 
A simple closed-loop active haptic guidance was used, consisting of guiding forces proportional to the user's deviation from the path $x_{\perp}$ and directed orthogonal to the path: $F_{G}=-k \cdot x_{\perp}$, with a factor $k=10 \mathrm{~N} / \mathrm{m}$.

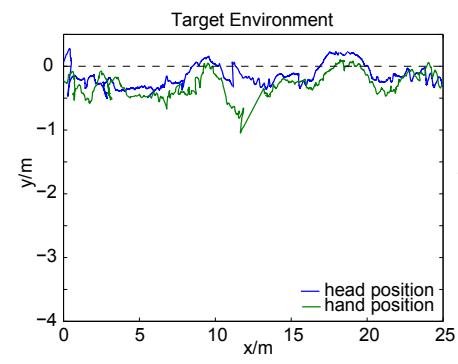

(a)

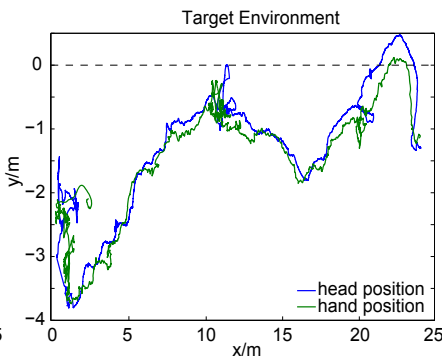

(b)
Fig. 5. Positions of user's head and user's hand in the target environment: (a) with closed-loop active haptic guidance, (b) without haptic guidance.

The experiment demonstrates the improved path-tracking with closed-loop haptic guidance in extended target environments. The maximal deviation from the path was $1 \mathrm{~m}$ with haptic guidance, whereas the deviation without haptic guidance was over $3 \mathrm{~m}$. Fig. 6(a) and Fig. 6(b) show a detail of the trajectory with closed-loop guidance in order to visualize the path covered by the user and the guiding forces in both environments during a test run.

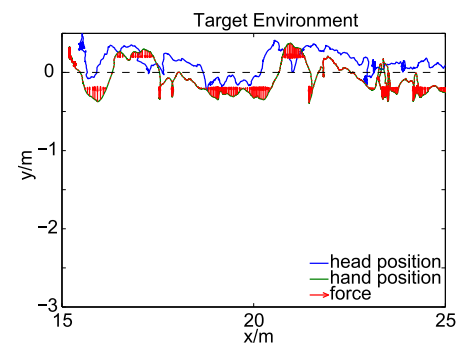

(a)

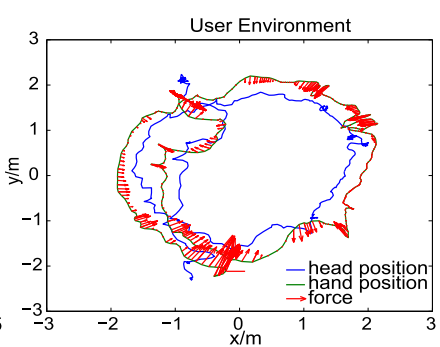

(b)
Fig. 6. Positions of user's head and user's hand, and guiding forces with closed-loop active haptic guidance: (a) in the target environment, (b) in the user environment.

\section{CONCLUSIONS}

This paper presents wide-area haptic guidance, a novel method for guiding an operator in telepresence scenarios of arbitrary size and shape. With active and passive force control, two complementary guidance methods for directing the user toward a desired direction are introduced. Both guidance methods can be combined in order to allow for a flexible guidance that may be adapted to any scenario.

Furthermore, two different path control modes were developed. Open-loop path control is purely goal-oriented and especially well-suited for guiding the operator toward a specific point without further influencing the actual path taken. In contrast, closed-loop path control seeks to keep the operator on a given path, which is used for avoiding certain obstacles or dangerous areas in the surroundings.

The guidance methods were implemented on a large haptic interface specifically designed for extended range telepresence. This haptic interface can display not only the high forces, required to realize hard guidance fixtures, but also a fine haptic rendering for soft guidance fixtures that do not degrade the user's immersion in the target environment. The large workspace of the haptic interface permits widearea haptic interaction and moreover, by employing Motion Compression, the user can be guided in arbitrarily large target environments. Experiments were performed in order to identify convenient guidance parameters for both, active and passive control methods, and to demonstrate the capability of closed-loop haptic guidance for compensating deviations along a large target path.

The proposed wide-area haptic guidance is not only adequate for teleoperation tasks that demand good speed and accuracy properties together with a high degree of immersion, but also for gaming and training applications that require the human navigation, like the training of escape routes and evacuations.

\section{REFERENCES}

[1] N. Nitzsche, U. D. Hanebeck, and G. Schmidt, "Motion Compression for Telepresent Walking in Large Target Environments," Presence: Teleoperators \& Virtual Environments, vol. 13, no. 1, pp. 44-60, Feb. 2004.

[2] P. Rößler, F. Beutler, U. D. Hanebeck, and N. Nitzsche, "Motion Compression Applied to Guidance of a Mobile Teleoperator," in Proceedings of the 2005 IEEE International Conference on Intelligent Robots and Systems (IROS 2005), Edmonton, Canada, Aug. 2005, pp. 2495-2500.

[3] A. Pérez Arias and U. D. Hanebeck, "A Novel Haptic Interface for Extended Range Telepresence: Control and Evaluation," in Proceedings of the 6th International Conference on Informatics in Control, Automation and Robotics (ICINCO 2009), Milan, Italy, July 2009, pp. 222-227.

[4] L. Rosenberg, "Virtual Fixtures: Perceptual Tools for Telerobotic Manipulation," in Proceedings of the IEEE Virtual Reality Interational Symposium, 1993, pp. 76-82.

[5] P. Marayong, A. Bettini, , and A. M. Okamura, "Effect of Virtual Fixture Compliance on Human-Machine Cooperative Manipulation," in Proceedings of the IEEE/RSJ International Conference on Intelligent Robots and Systems, 2002, pp. 1089-1095.

[6] D. Aarno, S. Ekvall, and D. Kragić, "Adaptive Virtual Fixtures for Machine-Assisted Teleoperation Tasks," in Proceedings of the IEEE International Conference on Robotics and Automation, 2005, pp. $1151-1156$.

[7] U. Unterhinninghofen, F. K. B. Freyberger, and M. Buss, "Study on Computer Assistance for Telepresent Reaching Movements," in Proceedings of the 6th International Conference on Haptics: Perception, Devices and Scenarios, 2008, pp. $745-754$.

[8] C. Weber, V. Nitsch, U. Unterhinninghofen, B. Färber, and M. Buss, "Position and Force Augmentation in a Telepresence System and Their Effects on Perceived Realism," in Proceedings of the Third Joint Eurohaptics Conference and Symposium on Haptic Interfaces for Virtual Environment and Teleoperator Systems, 2009, pp. 226-231.

[9] A. Bettini, P. Marayong, S. Lang, A. M. Okamura, and G. D. Hager, "Vision Assited Control for Manipulation Using Virtual Fixtures," IEEE Transactions on Robotics and Automation, vol. 20, no. 6, pp. 953-966, Dec. 2004.

[10] J. J. Abbott, P. Marayong, and A. M. Okamura, "Haptic Virtual Fixtures for Robot-Assisted Manipulation," in Proceedings of the 12th International Symposium Robotics Research, 2007, pp. 49-64.

[11] R. H. Taylor, P. Jensen, L. Whitcomb, A. Barnes, R. Kumar, D. Stoianovici, P. Gupta, Z. Wang, E. deJuan, and L. Kavoussi, "A Steady-Hand Robotic System for Microsurgical Augmentation," International Journal of Robotics Research, vol. 18, no. 12, pp. 12011210, Dec. 1999.

[12] C. A. Moore, M. A. Peshkin, and J. E. Colgate, "Cobot Implementation of Virtual Paths and 3D Virtual Surfaces," IEEE Transactions on Robotics and Automation, vol. 19, no. 2, pp. 347-351, Apr. 2003. 